\title{
Nanoscale Quantification of Interstitial Oxygen in Hyperstoichiometric $\mathrm{UO}_{2+\mathrm{x}}$
}

\author{
Steven R. Spurgeon ${ }^{1}$, Michel Sassi ${ }^{2}$, Colin Ophus ${ }^{3}$, Joanne Stubbs ${ }^{4}$, Eugene Ilton ${ }^{2}$, and Edgar Buck ${ }^{1}$ \\ 1. Energy and Environment Directorate, Pacific Northwest National Laboratory, Richland, USA \\ 2. Physical and Computational Sciences Directorate, Pacific Northwest National Laboratory, Richland, \\ USA \\ 3. The Molecular Foundry, Lawrence Berkeley National Laboratory, Berkeley, USA \\ 4. Center for Advanced Radiation Sources, University of Chicago, Chicago, USA
}

The safe use and disposal of oxide-based nuclear fuels depends on comprehensive models for oxidative processes, which can guide operation, long-term waste storage, and accident cleanup efforts. Because of their strategic importance and potential ecological impact, the oxidative behavior of the actinides (Th, $\mathrm{Pa}, \mathrm{U}, \mathrm{Np}, \mathrm{Pu}$ ) has been studied for many decades. These elements possess a complex $5 f$ electron configuration and exist in multiple valence states, leading to a range of exotic electronic and magnetic phase transitions that are influenced by oxygen defects. The oxidative behavior of the uraninite $\left(\mathrm{UO}_{2+\mathrm{x}}\right)$ system has received particular attention because of its use as a nuclear fuel, as well as its ability to readily adopt oxidation states spanning $\mathrm{U}^{4+} \rightarrow \mathrm{U}^{6+}$. The latter property has significant implications for the contamination of groundwater at waste storage sites, necessitating improved atomistic models for oxidation in ambient conditions.

To date, X-ray diffraction and spectroscopy studies have served as the primary means to investigate $\mathrm{UO}_{2}$ oxidation, yielding key insights into oxidative behavior. However, these techniques provide only volume-averaged information and typically require complex model fitting approaches. The development of aberration-corrected scanning transmission electron microscopy (STEM) allows us to directly probe structure, chemistry, and composition at the atomic-scale, offering a new method to examine actinide surfaces, interfaces, and grain boundaries.

Here we employ atomic-scale electron energy loss spectroscopy (EELS), interpreted in light of ab initio density functional theory (DFT) and electron channeling simulations, to examine over-oxidized model $\mathrm{UO}_{2+\mathrm{x}}$ single crystal surfaces. We compare (100) and (111)-oriented $\mathrm{UO}_{2+\mathrm{x}}$ samples previously measured using X-ray crystal truncation rod (CTR) analysis [1,2]. As shown in Figure 1, high-angle annular dark field (STEM-HAADF) imaging confirms that the fluorite structure is preserved to the top of the crystal, but significant changes in near-surface contrast point toward the incorporation of additional oxygen. EELS mapping of the surface, shown in Figure 2, reveals the emergence of key features in the $\mathrm{O} K$ edge that are only present in the over-oxidized band; DFT calculations show that these features result from changes in the interstitial content of the near-surface layers. Importantly, through the use of real-space mapping techniques, we are able to show that the fluorite structure is preserved to the topmost layer of the crystal, with no extended structural defects. We are able to fingerprint electronic fine structure changes that result from changes in interstitial oxygen content for the first time, opening a new route to directly interrogate the oxidative behaviour of $\mathrm{UO}_{2}$ and other actinides at the nanoscale. 


\section{References:}

[1] Stubbs, J. E. et al. Oxidative Corrosion of the $\mathrm{UO}_{2}$ (001) Surface by Nonclassical Diffusion. Langmuir 33, 13189-13196 (2017).

[2] Stubbs, J. E. et al. $\mathrm{UO}_{2}$ Oxidative Corrosion by Nonclassical Diffusion. Phys. Rev. Lett. 114, 246103 (2015).
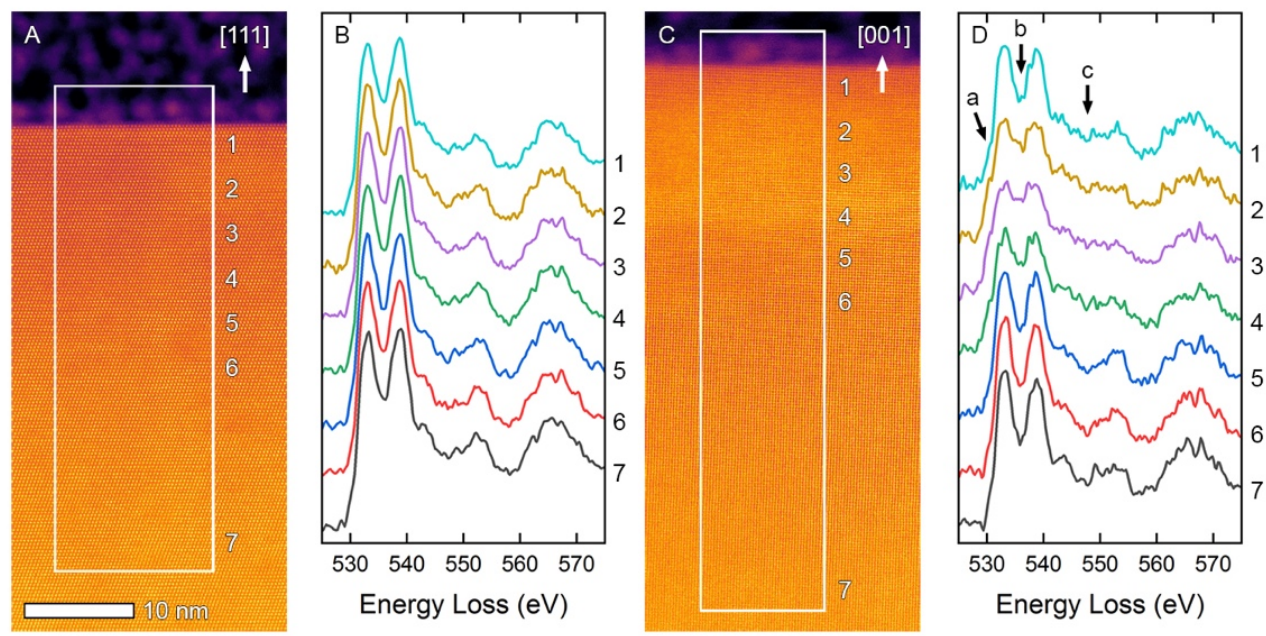

Figure 1. (A) STEM-HAADF image of the (111) surface and (B) corresponding O $K$ EEL spectra extracted from the numbered regions. (C) STEM-HAADF image of the (001) surface and (D) corresponding $\mathrm{O} K$ EEL spectra extracted from numbered regions. Emergent features are marked a-c.
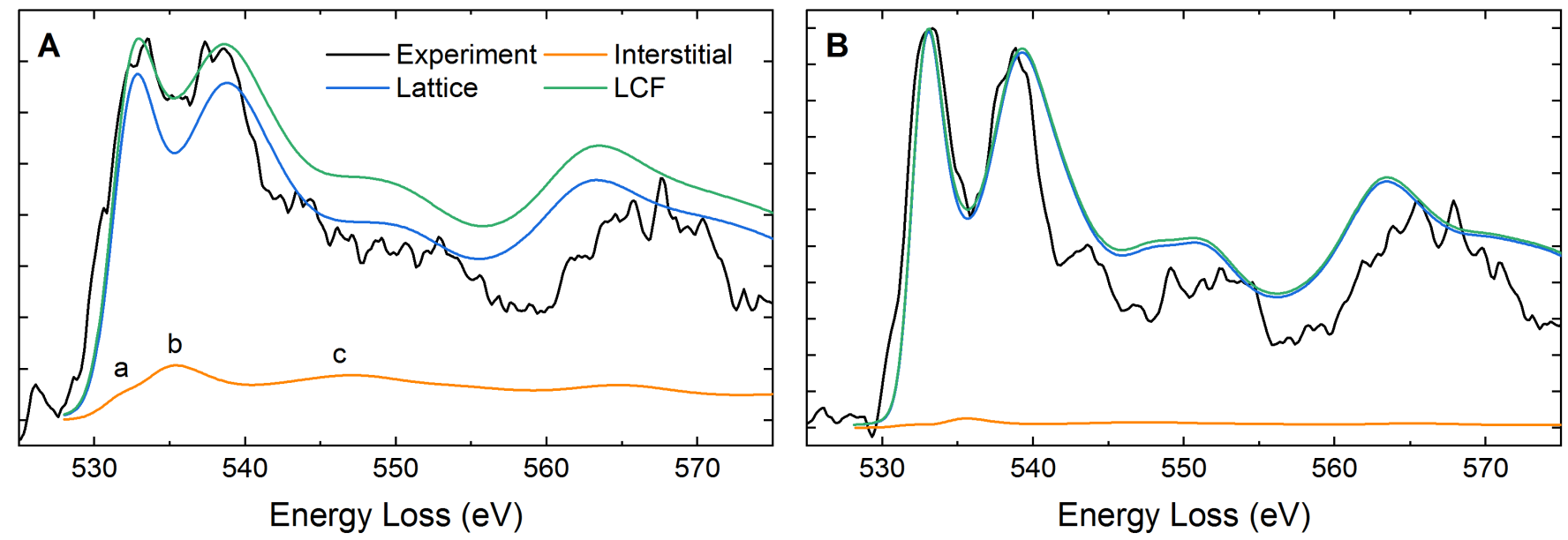

Figure 2. Comparison between experimental EEL and theoretically calculated X-ray absorption near edge (XANES) spectra accounting for the presence of interstitial oxygen. The individual contributions from $\mathrm{O}$ lattice sites, interstitial $\mathrm{O}$, and the resulting linear combinations are shown for spectrum 3 (A) and spectrum 7 (B) spectra taken from the (001) sample. Emergent features are marked by a-c. 